\title{
Determine the Role of FSH Receptor Binding Inhibitor in Regulating Ovarian Follicles Development and Expression of FSHR and ER $\alpha$ in Mice
}

\author{
Luju Lai, ${ }^{1}$ Xiaoyun Shen, ${ }^{2,3}$ Haoqin Liang, ${ }^{1}$ Yingying Deng, ${ }^{1}$ \\ Zhuandi Gong, ${ }^{4}$ and Suocheng Wei ${ }^{1}{ }^{1}$ \\ ${ }^{1}$ College of Life Science and Engineering, Northwest Minzu University, Lanzhou, Gansu 730030, China \\ ${ }^{2}$ State Engineering Technology Institute for Karst Desertification Control, Guizhou Normal University, Guiyang, \\ Guizhou 550001, China \\ ${ }^{3}$ School of Life Science and Engineering, Southwest University of Science and Technology, Mianyang, Sichuan 621010, China \\ ${ }^{4}$ Medicine College, Northwest Minzu University, Lanzhou, Gansu 730030, China \\ Correspondence should be addressed to Suocheng Wei; weisc668@163.com
}

Luju Lai and Xiaoyun Shen contributed equally to this work.

Received 23 December 2017; Accepted 14 June 2018; Published 9 July 2018

Academic Editor: Robert J. Lee

Copyright (C) 2018 Luju Lai et al. This is an open access article distributed under the Creative Commons Attribution License, which permits unrestricted use, distribution, and reproduction in any medium, provided the original work is properly cited.

\begin{abstract}
Mice of FRBI-1, FRBI-2, and FRBI-3 groups were intramuscularly injected with 20, 30, and 40mg/kg, respectively, for five consecutive days. Ovarian weights of three FRBI groups were reduced in comparison with FSH group. Ovarian cortex thicknesses (OCT) of the FRBI-3 group were less than that of the FSH group $(\mathrm{P}<0.05)$. As compared to FSH group, there were fewer numbers of secondary follicles (SFs) and mature follicles (MF) on the ovaries of FRBI-treated mice numbers of primary follicles (PFs) and SFs also decreased. In FRBI-3 mice, we found that the primordial follicles (POF) were scarcer, the follicles developed poorly, and granulosa cells became apoptosis. SF numbers of FRBI-2 and FRBI-3 groups were less than that of the FSH group on day 20 $(\mathrm{P}<0.05)$. Maximum longitudinal diameter $(\mathrm{MLD})$ and transverse diameter (MTD) of three FRBI groups became decreased during the experiment. MLD and MTD of the FRBI-3 group were smaller than FSH group. Levels of FSHR mRNA and protein were less than that of CG and FSH group $(\mathrm{P}<0.05)$. ER $\alpha$ protein levels of FRBI group and serum concentrations of $F S H$ and estradiol $\left(\mathrm{E}_{2}\right)$ in the FRBI-treated mice were decreased when compared to CG and FSH group. In conclusion, FSH treatment could increase the numbers of SF and MF, enhance follicle development, reduce the numbers of SF and MF, and depress the follicular development of mice. Furthermore, FRBI declined the mRNA and protein levels of ER $\alpha$ and FSHR in the ovaries and dropped serum concentrations of $\mathrm{FSH}$ and $\mathrm{E}_{2}$ of mice.
\end{abstract}

\section{Introduction}

Follicle stimulating hormone $(\mathrm{FSH})$ and estradiol $\left(\mathrm{E}_{2}\right)$ can precisely regulate the female fertility depending on the development of ovarian follicles and final ovulation $[1,2]$. The interaction between FSH and its cognate receptor (FSHR) activates multiple signaling pathways leading to steroidogenesis production that modulates the differentiation and proliferation of ovarian granulosa cells [3]. FSHR activates the extracellular signal-regulated kinases (ERK). However, the mechanisms of these actions are unknown [4].
FSH receptor binding inhibitor (FRBI) blocked the combination of FSH into FSHR and inhibited FSH action on at the gene and protein levels $[5,6]$. In vivo administration of FRBI resulted in the suppression of ovulation and induced follicular atresia in mice [7] and impacted the fertility in marmosets [8]. Recently, there has been little information about FRBI effects on follicular development and reproduction functions in human and animals [3, 9]. The exact mechanism of FRBI actions remains still unclear $[3,10]$. 
Estrogen regulates fertility of human and animals. Cellular responses to estrogen are mediated by estrogen receptor $\alpha$ $(\mathrm{ER} \alpha)$ and estrogen receptor $\beta(\mathrm{ER} \beta)$ [11].

The binding of estrogen to its receptors (ERs) interacts with nuclear estrogen response elements leading to transcription initiation [12]. In mouse and rat models, disruption of $\mathrm{ER} \alpha$ causes infertility in both males and females. However, the roles of $\mathrm{ER} \alpha$ and $\mathrm{ER} \beta$ in reproductive function remain undecided [13]. Up to date, it remains unclear if FRBI treatment impacts the expression levels of estrogen receptors in the ovarian follicles $[14,15]$.

The present work was performed to assess the effects of FSH receptor binding inhibitor (FRBI) on the development of ovaries and follicles and reproduction functions, to understand the FRBI mechanism of inhibiting the interaction of FSH to FSHR in the follicles, and to investigate the signal transduction and pathway of FRBI actions in mice.

\section{Materials and Methods}

2.1. Preparation of FSH Receptor Binding Inhibitor (FRBI). The FSH receptor binding inhibitor (FRBI) peptide of $99.9 \%$ purity was synthesized and characterized before being used for the experiments. The preparation of FRBI was performed according to the methods established in our laboratory $[4,8]$. The concentration of FRBI was $1000 \mu \mathrm{g} / \mathrm{mL}$.

2.2. Animal Treatment. 150 Kunming female mice, 21 days old, body weight of $18.00 \pm 1.23 \mathrm{~g}$, were purchased from Lanzhou University [License No. SCXK (Gansu) 2005-0007]. All mice were randomly allocated to FRBI group, FSH group, and control group (CG) $(n=30)$. FRBI of 20, 30, and $40 \mathrm{mg} / \mathrm{kg}$ body weight were intramuscularly injected into the mice of FRBI-1, FRBI-2, and FRBI-3, respectively, for five consecutive days. 10IU FSH was intramuscularly injected into mice of FSH group for five consecutive days. $0.2 \mathrm{~mL}$ saline was injected into mice of CG for five consecutive days. Injections were made in the morning (at 8 to 9 a.m.) each day. Referring to our previous procedure [16], all mice were raised in the group, kept in mice cages, and accurately weighed each day using an electronic balance.

2.3. Sample Collections and Measurements. After five mice from each group were injected intramuscularly $0.1 \mathrm{mg} / \mathrm{kg}$ xylazine on days $0,7,10,15,20$, and 30 , respectively, they were killed by cervical dislocation. Bilateral ovaries were aseptically cut using a sharp scalpel. The weight of each ovary was weighed immediately on an electronic balance. The average value was calculated based on bilateral ovaries of each mouse. Meanwhile, blood samples were harvested on days 0 , $7,10,15,20$, and 30, respectively. Serum was separated and stored at $-20^{\circ} \mathrm{C}$.

2.4. Histological Observations and Measurement of Ovaries and Follicles. Ovaries were fixed in 10\% formaldehyde, embedded with paraffin wax, then sliced $(5 \mu \mathrm{m})$, and finally stained with hematoxylin and eosin (H\&E). The sections were observed under the light microscope (Leica, Japan). Secondary follicles (3-5 $\mathrm{mm}$ in diameter) were found and counted. Microscopic images of the ovaries were photographed. Six sites in each section (5 sections in every group, totaling 150 sites for each group) were measured. The ovarian cortex thickness, maximum transverse diameter (MTD) (MLD), and longitudinal diameter of each secondary follicle were measured, respectively, using Pro Plus 2.0 (MOTIC Company, Hong Kong, China).

2.5. Real Time RT-PCR ( $q R T-P C R)$ of FSHR and ER $\alpha$ mRNAs. The levels of ER $\alpha$ and FSHR mRNAs were determined using real time fluorescence quantitative PCR (qRT-PCR) and cloning techniques, so as to evaluate the FRBI effects on expressions of ER $\alpha$ and FSHR mRNAs in mouse ovaries.

2.5.1. Primer Design. The primers specific for ER $\alpha$ (NM001302531.1) and FSHR (GenBank accession number: NM013523.3) were designed with Beacon Designer 7.0 software (Premier Biosoft International, Palo Alto, CA, USA) according to manufacturer's guidelines and Primer-BLAST at NCBI. The reference gene was mouse GAPDH gene (NM008084.2, HM-043737.1) which was used for normalizing expression levels of target genes $[17,18]$. The sequences of the primers used in the qPCR were as follows: FSHR, forward $5^{\prime}$-CGTCCTGATGAGCAAGTTTGG-3' and reverse, $5^{\prime}$ TGGGCTGATTGACTTAGAGGG-3'; ER $\alpha$, forward $5^{\prime}$ CTTGTGTGTGGACACTCCGT- $3^{\prime}$ and reverse, $5^{\prime}$-AAGAAAGGCACAAGGCACGA- $3^{\prime}$; GAPDH, forward, $5^{\prime}$-CTTCAACAGCGACACTCACTCT- $3^{\prime}$ and reverse, $5^{\prime}$-CCACCACCCTGTTGCTGTA-3'.

The concentrations of the primers $(100 \mathrm{nM}, 200 \mathrm{nM}, 300$ $\mathrm{nM}$, and $500 \mathrm{nM}$ ) were evaluated, and primer-dimer formation was determined using the melting curve analysis. The primer concentrations only showed dimmer-free reactions were used for the further experiment.

2.5.2. RNA Extraction and cDNA Synthesis. In accordance with the manufacturer's instructions $[17,18]$, the total RNA was extracted from the ovarian samples using the Trizol reagent (Invitrogen, Beijing, China).

The cDNA was synthesized with the superscript ${ }^{\mathrm{TM}}$ firststrand synthesis system for the reverse transcription PCR (RT-PCR) (Invitrogen, Beijing, China).

2.5.3. Fluorescence Quantitative RT-PCR ( $q P C R)$. The expression levels of $\mathrm{ER} \alpha$ and FSHR mRNAs were determined using qPCR based on our previous methods $[4,17]$. The relative level of each mRNA was calculated with the $2^{-\Delta \Delta} \mathrm{Ct}$ method and normalized to GAPDH gene on day 0 . The samples were detected in triplicate.

2.6. Western Blots of ER $\alpha$ and FSHR Proteins in Mouse Ovaries. Western blots were carried out referring to our laboratory methods [19]. The integral optical density (IOD) of the scanned bands was achieved by using Quantity One software (Bio-Rad Company, Hercules, CA, USA). A negative control was performed without primary antibody. The relative contents of ER $\alpha$ and FSHR proteins were expressed as the proportion between gray values of ER $\alpha$ and FSHR proteins divided by that of $\beta$-actin. 


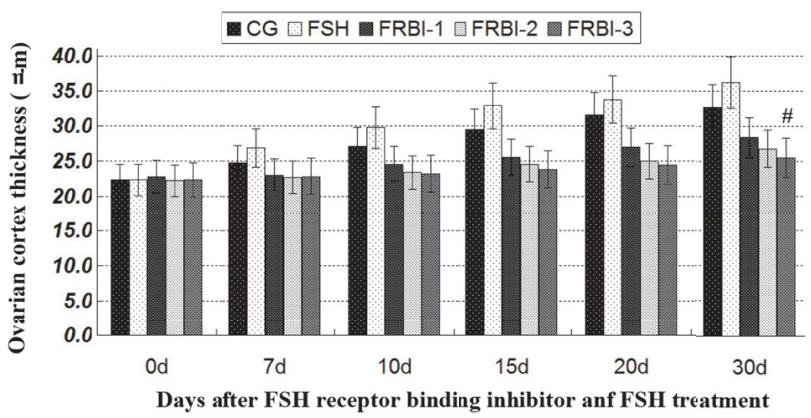

FIgure 1: Ovarian cortex thickness of mice. OCTs of three FRBI groups were decreased as compared to CG and FSH group. OCT of the FRBI-3 group was less than that of the FSH group on day 30. ${ }^{\#} \mathrm{P}<0.05$ as compared to FSH group.

2.7. Detection of Serum FSH and $E_{2}$ Concentrations. Serum concentrations of estradiol $\left(\mathrm{E}_{2}\right)$ and $\mathrm{FSH}$ were detected with the especial $\mathrm{E}_{2}$ and $\mathrm{FSH}$ kits for mice (ELISA) following the operation manual (Cusabio Biotech Co., Ltd., Wuhan, China), respectively. Detection limits were $0.02 \mathrm{pg} / \mathrm{mL}$ for $\mathrm{E}_{2}$ and $0.01 \mathrm{ng} / \mathrm{mL}$ for FSH. The inter- and intra- experimental variation coefficients were less than $6 \%$ and $5 \%$. All samples were tested in triplicate. The detailed methods were reported in our earlier study [17].

2.8. Data Statistical Analyses. For each group, all parameters (including OCT, MLD, MTD, levels of ER $\beta$ and FSHR, serum $\mathrm{E}_{2}$ and $\mathrm{FSH}$ ) were calculated on the basis of the data of 5 mice in each subgroup, respectively, using SPSS v. 21.0 (SPSS Inc. Chicago, IL, USA). Data are expressed as means \pm SEM. Data from each time point was analyzed separately. All variables of five groups complied with the assumptions for a oneway ANOVA. Supplementary Tukey's post hoc tests were done to determine the pairwise differences after significant differences were identified. The significant level was $\mathrm{P}<0.05$.

\section{Results}

3.1. Ovarian Weights and Cortex Thickness of Mice. In order to assess the effects of FRBI administration on ovarian development, the ovarian weight and ovarian cortex thickness (OCT) were detected, respectively. In comparison to the control group (CG). Ovarian weights of FRBI groups were dose-dependently lower than that of FSH-treated group (data omitted). But there was no significant difference between groups.

As shown in Figure 1, OCT of FSH group was slightly increased as compared to CG. OCTs of FRBI groups were reduced in comparison with $\mathrm{CG}$ and FSH group, with a minimum value of the FRBI-3 group. On day 30, OCT of FRBI-3 group was less than that of the FSH group $(\mathrm{P}<0.05)$. The results demonstrated that a high dose of FBRI $(40 \mathrm{mg} / \mathrm{kg})$ could reduce OCT of mice.

3.2. Histology Structures of Follicles. To determine effects of FRBI regulating FSH function on follicular development, ovarian structure was observed under microscope $(\times 100)$. Histological changes on day 20 were described in all groups as follows.

3.2.1. Control Group (CG). The primordial follicles (POF) and primary follicles (PF) were small. A few of the secondary follicles (SF) and mature follicles (MF) existed. The structures of the ovaries and follicles were complete. Ovarian cortex and zona pellucida (ZP) were distinct (Figure 2(a)).

3.2.2. FSH Group. The rich SFs and MFs were distributed. POF and PF became larger, and SF numbers were obviously increased in comparison with CG (Figure 2(b)). The follicular antrum was formed in MFs. Dense granulosa cell layers distributed over SF. Follicles developed fully.

3.2.3. FRBI Groups. The ovaries and follicles were structured completely. Fewer numbers of SF and MF existed on the ovaries in comparison with FSH group. The granulosa cells of SFs arranged tightly (Figure 2(c)). In FRBI-2 group, few SF and MF distributed. PF and SF numbers were reduced. Zona pellucida (ZP) became small. Follicles developed poorly. The granulosa cells were found to become apoptosis (Figure 2(d)). POFs were rarer than that of FRBI-3. Scarce SFs and MFs were observed as compared to FSH and CG groups. Follicles developed poorly. The apoptosis of granulosa cells was observed in FRBI-3 group (Figure 2(e)).

These results demonstrated that FSH treatment could increase the numbers of SF and MF, thus enhancing follicle development. FRBI administration reduced the numbers of SF and MF and therefore suppressed the follicle development.

3.3. Numbers of Secondary Follicles in Mice. Data in Table 1 showed that numbers of secondary follicles of FSH group were greater than that of CG during the experiment, with the highest increment found on day $20(\mathrm{P}<0.01)$. However, follicles numbers of FRBI groups were decreased from day 7 when compared to CG and FSH group. On day 20, follicle numbers of three FRBI groups were less than that of the FSH group $(\mathrm{P}<0.01)$. In addition, follicle numbers of the FRBI-3 group were lower than CG on days 15, 20, and 30 . The findings indicated that FSH promoted follicular development. FRBI administration decreased the numbers of secondary follicles and depressed the follicular development of mice.

3.4. MLD and MTD of Secondary Follicles. In FSH group, MLD and MTD were increased in comparison to CG (Figure 3). On the contrary, MLD and MTD of three FRBI groups were decreased during the experiment. On day 30, MLD and MTD of FRBI-3 group were significantly lower than those of FSH. The results indicated that FRBI administration could suppress the follicle development of mice.

3.5. Expression Levels of ER $\alpha$ and FSHR mRNAs in Ovaries on Day 30. As shown in Figure 4, ER $\alpha$ and FSHR were expressed in mouse ovaries. Expression levels of $\operatorname{ER} \alpha$ and FSHR mRNAs were gradually declined in a dose-dependent model. In FRBI-2 and FRBI-3 groups, levels of FSHR mRNAs were lower than CG $(\mathrm{P}<0.05)$ and $\mathrm{FSH}$ group $(\mathrm{P}<0.01)$ on 


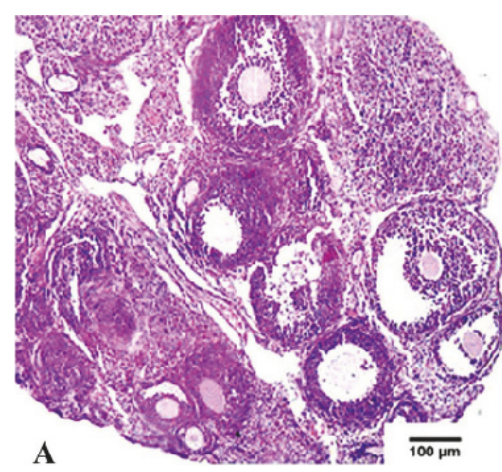

(a)

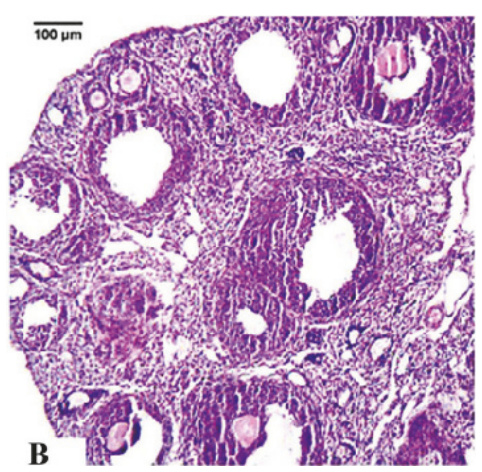

(b)

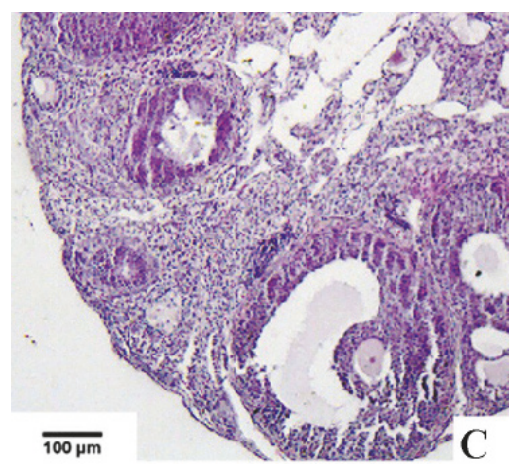

(c)

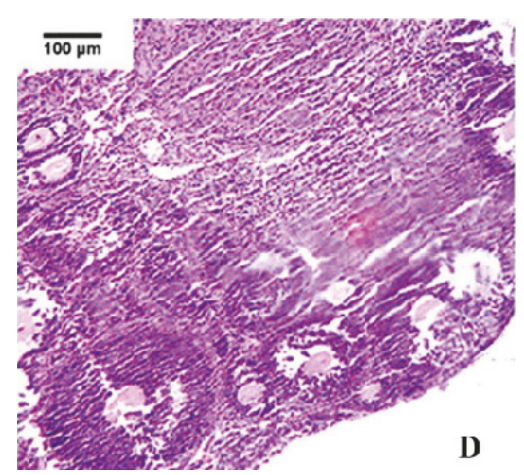

(d)

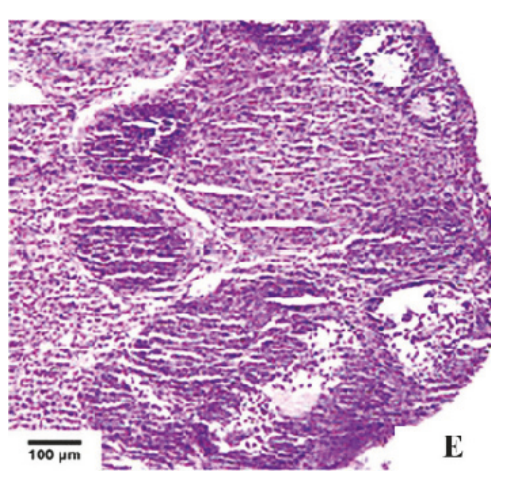

(e)

FIGURE 2: Histological observation of ovaries and follicles on day $20(\times 100$, scale bar $=10 \mu \mathrm{m})$. (a) Control group (CG); (b) FSH group; (c) FRBI-1 group; (d) FRBI-2 group; and (e) FRBI-3 group.

TABLE 1: Numbers of secondary follicles $(\times 400)$.

\begin{tabular}{|c|c|c|c|c|c|c|}
\hline Group & $0 \mathrm{~d}$ & $7 \mathrm{~d}$ & $10 \mathrm{~d}$ & $15 \mathrm{~d}$ & $20 \mathrm{~d}$ & $30 \mathrm{~d}$ \\
\hline CG & $3.83 \pm 0.38$ & $4.50 \pm 0.42$ & $4.96 \pm 0.43$ & $5.21 \pm 0.49$ & $5.65 \pm 0.51$ & $6.50 \pm 0.59$ \\
\hline FSH & $3.84 \pm 0.33$ & $5.64 \pm 0.56$ & $6.27 \pm 0.59$ & $7.01 \pm 0.63$ & $9.50 \pm 0.52^{* *}$ & $7.63 \pm 0.62$ \\
\hline FRBI-1 & $3.81 \pm 0.35$ & $3.50 \pm 0.40$ & $3.23 \pm 0.28^{\#}$ & $4.02 \pm 0.39^{\#}$ & $4.69 \pm 0.38^{\# \#}$ & $5.06 \pm 0.46$ \\
\hline FRBI-2 & $3.82 \pm 0.35$ & $3.12 \pm 0.32^{* \#}$ & $3.65 \pm 0.38$ & $3.11 \pm 0.31^{* \# \#}$ & $4.38 \pm 0.36^{\# \#}$ & $4.55 \pm 0.39^{\#}$ \\
\hline FRBI-3 & $3.81 \pm 0.32$ & $2.53 \pm 0.37^{* \# \#}$ & $3.15 \pm 0.27^{\#}$ & $3.04 \pm 0.28^{* \# \#}$ & $4.32 \pm 0.41^{\text {*\#\# }}$ & $4.11 \pm 0.40^{* \#}$ \\
\hline
\end{tabular}

Note: ${ }^{*} \mathrm{P}<0.05$ as compared to $\mathrm{CG} ;{ }^{* *} \mathrm{P}<0.01$ as compared to $\mathrm{CG}$;

${ }^{\#} \mathrm{P}<0.05$ as compared to FSH group; and ${ }^{\# \#} \mathrm{P}<0.05$ as compared to FSH group.

day 30. The findings in our work demonstrated that FRBI treatment reduced FSHR mRNA expression in the ovaries.

3.6. Levels of FSHR and ER $\alpha$ Proteins in Ovaries. FSHR and $\mathrm{ER} \alpha$ protein levels were gradually increased in FSHtreated mice from day 7 afterwards of the first FSH treatment (Figure 5). FSHR and ER $\alpha$ protein levels of three FRBI groups were reduced in comparison with CG (ER $\alpha$ data omitted). FSHR protein levels of FRBI groups were significantly decreased as compared to CG on day $30(\mathrm{P}<0.05)$ and $\mathrm{FSH}$ group on days 20 and $30(\mathrm{P}<0.01)$. ER $\alpha$ protein levels of FRBI group were also less than the FSH group on day $30(\mathrm{P}<0.05)$. The results exhibited that administration of FRBI suppressed expression of $\mathrm{ER} \alpha$ and FSHR protein in mouse ovaries.

3.7. Serum Concentrations of FSH and Estradiol $\left(E_{2}\right)$. Serum $\mathrm{FSH}$ concentrations of all mice increased gradually within the experiment. Serum FSH concentration of FSH-treated mice was higher than that of CG $(\mathrm{P}<0.05)$ from days 15 (Table 2$)$. FSH levels of three FRBI groups decreased when compared to CG and FSH group. On days 20 and 30, FSH levels of FSH group were higher than that of FRBI-1 $(\mathrm{P}<0.05)$ and those of FRBI-2 and FRBI-3 $(\mathrm{P}<0.01)$. The findings demonstrated that FRBI could attenuate serum FSH concentrations.

As shown in Table 3, $\mathrm{E}_{2}$ concentrations of three FRBI groups were lower than that of FSH group. There was a significant reduction of $\mathrm{E}_{2}$ concentrations of FRBI- 2 and FRBI-3 on days 20 and 30. The findings revealed that FRBI could decline secretion of FSH and $\mathrm{E}_{2}$ of mice.

\section{Discussion}

The development and growth of ovarian follicles are precisely regulated by many genes, such as FSH [2, 20, 21]. FSH acts 
TABLE 2: Serum FSH concentrations (ng/mL).

\begin{tabular}{lcccccc}
\hline Group & 0d & $7 \mathrm{~d}$ & $10 \mathrm{~d}$ & $15 \mathrm{~d}$ & $20 \mathrm{~d}$ & $30 \mathrm{~d}$ \\
\hline CG & $9.74 \pm 1.31$ & $12.24 \pm 1.14$ & $15.76 \pm 1.68$ & $18.67 \pm 1.71$ & $22.56 \pm 2.23$ & $26.88 \pm 2.42$ \\
FSH & $9.54 \pm 1.21$ & $15.44 \pm 1.24$ & $21.12 \pm 1.88$ & $30.35 \pm 3.72^{*}$ & $39.88 \pm 4.56^{*}$ & $45.78 \pm 5.73^{* *}$ \\
FRBI-1 & $9.82 \pm 1.01$ & $11.68 \pm 1.19$ & $19.12 \pm 1.79$ & $18.6 \pm 1.91$ & $21.27 \pm 2.32^{\#}$ & $30.02 \pm 3.42^{\#}$ \\
FRBI-2 & $9.58 \pm 1.09$ & $9.05 \pm 1.28$ & $12.24 \pm 1.18^{\#}$ & $13.15 \pm 1.13^{\#}$ & $15.78 \pm 1.48^{* \# \#}$ & $20.63 \pm 2.21^{\# \#}$ \\
FRBI-3 & $9.69 \pm 1.02$ & $8.08 \pm 1.01^{\#}$ & $7.88 \pm 0.77^{\# \#}$ & $9.47 \pm 1.34^{\# \#}$ & $12.28 \pm 1.16^{* \# \#}$ & $18.64 \pm 1.95^{* \# \#}$ \\
\hline
\end{tabular}

Note: ${ }^{*} \mathrm{P}<0.05$ as compared to $\mathrm{CG} ;{ }^{* *} \mathrm{P}<0.01$ as compared to $\mathrm{CG}$;

${ }^{\#} \mathrm{P}<0.05$ as compared to FSH group; and ${ }^{\# \#} \mathrm{P}<0.05$ as compared to FSH group.

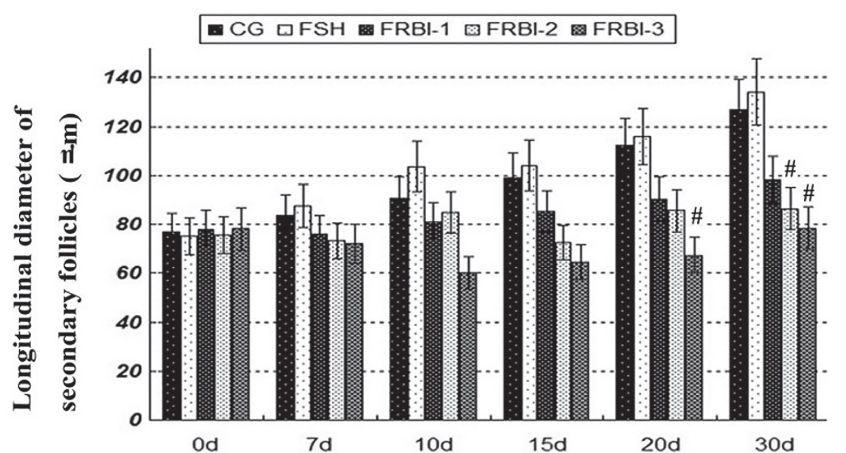

Days after FSH receptor-binding inhibitor and FHS treatment

(a)

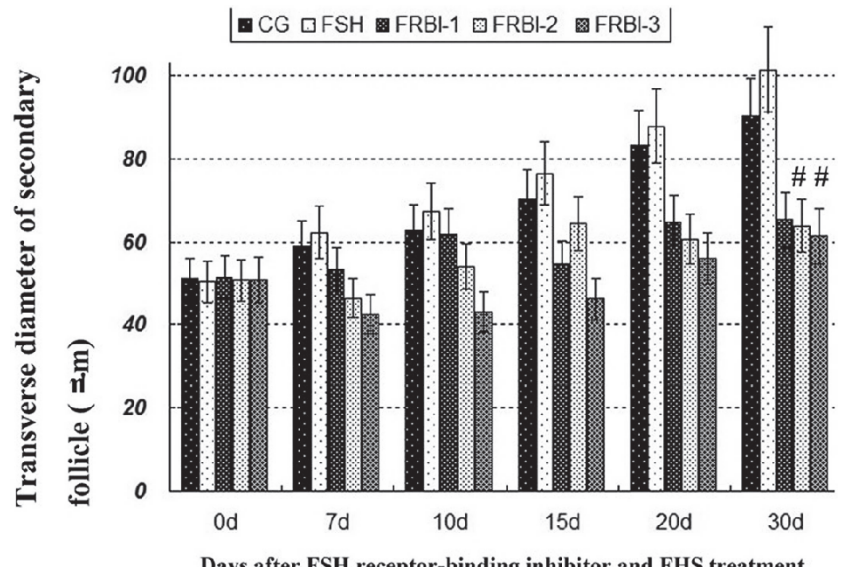

(b)

Figure 3: MLD and MTD of mice secondary follicles $(\times 400$, $\mu \mathrm{m})$. (a) Maximum longitudinal diameter (MLD). (b) Maximum transverse diameter (MTD). ${ }^{\#} \mathrm{P}<0.05$ as compared to $\mathrm{FSH}$ group.

via cognate FSHR that is mainly expressed by granulosa cells in the follicles $[6,21]$. Blocking FSH-FSHR interaction resulted in the decline of FSH action. FSH receptor binding inhibitor (FRBI), as an FSH antagonist, suppressed FSHFSHR interaction and therefore influenced the efficacy of FSH $[5,22]$. In vivo administration of FRBI resulted in the suppression of ovulation and induced follicular atresia and apoptosis of mice $[7,22]$ and further impaired the proliferation of granulosa cells [6]. In the present work, the female mice were treated with varying doses of FRBI in order to assess FRBI effects on ovarian and follicular

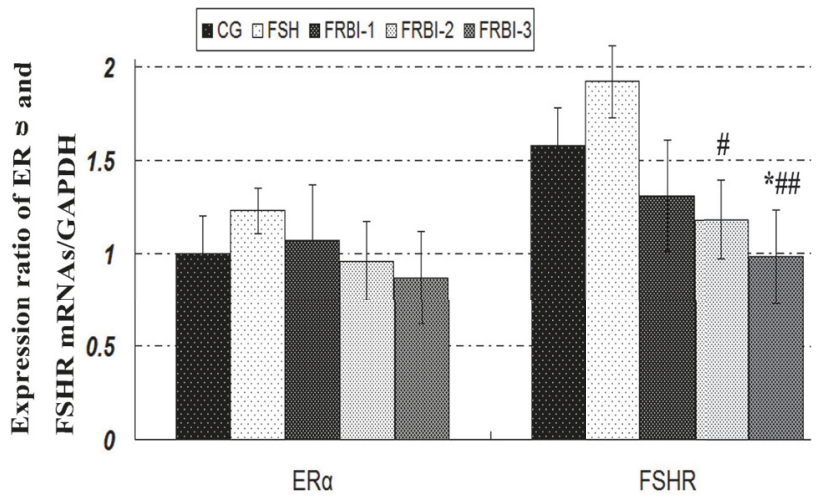

FIGURE 4: Expression levels of ER $\alpha$ and FSHR mRNAs. Levels of FSHR mRNAs were lower than that of CG and FSH group on day 30 after the first FRBI injection. ${ }^{*} \mathrm{P}<0.05$ as compared to $\mathrm{CG}$; ${ }^{*} \mathrm{P}<0.05$ as compared to FSH group. ${ }^{\# \#} \mathrm{P}<0.05$ as compared to FSH group.

development. FRBI slightly decreased ovary weights, ovarian cortex thickness (OCT), and numbers of ovarian secondary follicles (SF) and mature follicles (MF) on the ovaries. The primordial follicles (POF) and primary follicles (PF) were scarcer. MLD and MTD values of FRBI groups were reduced during the experiment. Follicles developed poorly in the FRBI-treated mice. In vivo administration of FRBI depressed the follicle development of mice. Our outcomes were in accordance with previous documents $[19,22]$. However, little information has been reported regarding FRBI influences on follicular development in human and animals [3, 10]. The findings in our work still need to be further investigated in the future. The mechanism of FRBI actions also needs thoroughly exploring.

FRBI, a nonsteroidal low molecular weight factor, not only blocked the binding of FSH to FSHR [6], but also altered FSH action of rat granulosa cells at the receptor level $[6,8]$. The progesterone $(\mathrm{P})$ secretion was dose-dependently suppressed after the granulosa cells of rats were treated with FRBI in the presence or absence of FSH [6]. FRBI addition into the in vitro maturation medium could decrease the expression levels of FSHR and LHR mRNAs and proteins in cumulus-oocyte complexes (COCs) of sheep [19]. The results in this study indicated that FRBI injection could decline the expression levels of ER $\alpha$ and FSHR mRNAs and proteins in the ovaries of mice. The findings were consistent with previous documents $[6,19]$. Our findings were in 
TABLE 3: Serum estradiol $\left(\mathrm{E}_{2}\right)$ concentrations $(\mathrm{pg} / \mathrm{mL})$.

\begin{tabular}{lcccccc}
\hline Group & $0 \mathrm{~d}$ & $7 \mathrm{~d}$ & $10 \mathrm{~d}$ & $15 \mathrm{~d}$ & $20 \mathrm{~d}$ & $30 \mathrm{~d}$ \\
\hline CG & $97.6 \pm 10.3$ & $107.4 \pm 11.5$ & $113.1 \pm 12.4$ & $117.4 \pm 10.6$ & $120.8 \pm 13.8$ & $125.8 \pm 12.7$ \\
FSH & $98.6 \pm 9.8$ & $110.6 \pm 10.6$ & $119.4 \pm 11.3$ & $123.2 \pm 12.0$ & $135.6 \pm 12.7$ & $146.2 \pm 15.2^{*}$ \\
FRBI-1 & $96.7 \pm 9.6$ & $94.1 \pm 8.9$ & $92.4 \pm 10.2^{\#}$ & $98.2 \pm 10.8^{* \#}$ & $102.3 \pm 11.1$ & $103.3 \pm 11.2^{\# \#}$ \\
FRBI-2 & $98.2 \pm 10.1$ & $90.2 \pm 9.2$ & $80.2 \pm 9.1^{\text {*\# }}$ & $93.8 \pm 8.5^{\text {*\# }}$ & $99.6 \pm 9.6^{* \# \#}$ & $117.5 \pm 10.3^{\#}$ \\
FRBI-3 & $98.7 \pm 9.9$ & $86.0 \pm 7.5$ & $72.6 \pm 7.8^{\text {*\# }}$ & $88.6 \pm 9.1^{\text {*\# }}$ & $96.4 \pm 10.2^{\text {*\#\# }}$ & $104.6 \pm 10.5^{\# \#}$ \\
\hline
\end{tabular}

Note: ${ }^{*} \mathrm{P}<0.05$ as compared to $\mathrm{CG} ;{ }^{\#} \mathrm{P}<0.05$ as compared to FSH group; and

\#\# $\mathrm{P}<0.05$ as compared to FSH group.
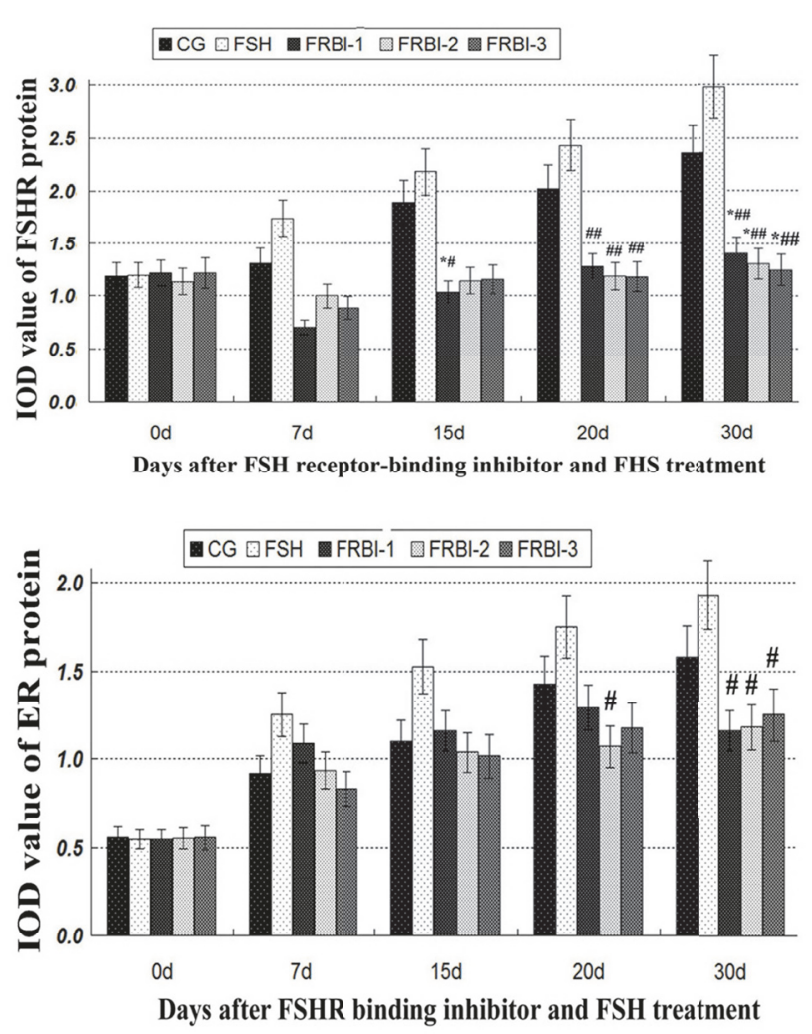

FIGURE 5: Expression levels of ER $\alpha$ and FSHR proteins in the ovaries. $\mathrm{ER} \alpha$ and FSHR protein levels of FRBI groups were decreased when compared to CG. ${ }^{*} \mathrm{P}<0.05$ as compared to $\mathrm{CG} ;{ }^{\#} \mathrm{P}<0.05$ as compared to $\mathrm{FSH}$ group. ${ }^{\# \#} \mathrm{P}<0.05$ as compared to $\mathrm{FSH}$ group.

agreement with initial studies $[23,24]$. These were probably that FRBI blocked the interaction between FSH and FSHR and, therefore, influenced the efficacy of FSH $[5,6]$ because FRBI impaired FSH-FSHR combination at the receptor level [22]. The mechanism of FRBI actions has to be thoroughly explored in other animals and humans.

Estradiol $\left(\mathrm{E}_{2}\right)$ is a main circulating estrogen hormone [11]. $\mathrm{E}_{2}$ activates the growing large ovarian follicles and promotes follicular growth and differentiation [25]. $\mathrm{E}_{2}$ protects granulosa cells from apoptosis and promotes cell cycle progression of healthy follicles [26]. FRBI treatment inhibited progesterone secretion and hampered the growth and differentiation of granulosa cells $[8,22]$. Our previous studies indicated that FRBI treatment could suppress FSH production of sheep COCs [19]. The findings in this study showed the serum FSH concentration of FSH-treated mice was higher than that of CG from days 15 . But, FSH and $\mathrm{E}_{2}$ concentrations of FRBI groups were decreased as compared to CG and FSH group. Therefore, FRBI impacted FSH and $\mathrm{E}_{2}$ secretion of mice, which influenced fertility of animals. Our findings were similar to previous document [22, 23, 27]. But they were a disagreement with our initial study in sheep COCs [4]. The actual action of FRBI effects on endocrine function still remains unclear $[10,28]$ and needs to be investigated.

\section{Conclusions}

FSH treatment could increase the numbers of SF and MF, thus enhancing follicle development. FRBI administration reduced the numbers of SF and MF and depressed the follicular development of mice. A high dose of FBRI $(40 \mathrm{mg} / \mathrm{kg}$ ) could reduce OCT of mice. Furthermore, FRBI could drop the mRNA and protein levels of ER $\alpha$ and FSHR in the mouse ovaries and decline serum concentrations of FSH and $\mathrm{E}_{2}$ of mice. Our study offered a solid basis for thoroughly elucidating the mechanism of FRBI. These will be conducive to promoting ovarian and follicular functions and further to enhance animal's fertility.

\section{Data Availability}

The datasets used and analyzed in the present investigation are available from the corresponding author on reasonable request.

\section{Ethical Approval}

All procedures regarding animal treatment were approved by the Experiment Animal Care and Use Committee of Gansu Province, China.

\section{Conflicts of Interest}

The authors declare no conflicts of interest.

\section{Authors' Contributions}

Dr. Luju Lai and Xiaoyun Shen contributed equally to this work. 


\section{Acknowledgments}

This work was supported by the State Key Research and Development Program in 13th Five-Year of China (Grant no. 2016 YFC0502601), the Reproductive Biotechnology Innovation Team of Animals (Grant no. 2017C-01) of Colleges and Universities of Gansu Province, and the National Natural Science Foundation of the People's Republic of China (Grants nos. 41671041 and 31460684).

\section{References}

[1] S. L. Field, T. Dasgupta, M. Cummings, and N. M. Orsi, "Cytokines in ovarian folliculogenesis, oocyte maturation and luteinisation," Molecular Reproduction and Development, vol. 81, no. 4, pp. 284-314, 2014.

[2] H. Xu, K. Deng, Q. Luo et al., "High serum FSH is associated with brown oocyte formation and a lower pregnacy rate in human IVF parctice," Cellular Physiology and Biochemistry, vol. 39, no. 2, pp. 677-684, 2016.

[3] E. M. Donaubauer and M. E. Hunzicker-Dunn, "Extracellular Signal-regulated Kinase (ERK)-dependent phosphorylation of Y-Box-binding protein 1 (YB-1) enhances gene expression in granulosa cells in response to follicle-stimulating hormone (FSH)," The Journal of Biological Chemistry, vol. 291, no. 23, pp. 12145-12160, 2016.

[4] G. Zhuandi, L. Haoqin, D. Yingying et al., "FSH receptor binding inhibitor influences estrogen production, receptor expression and signal pathway during in vitro maturation of sheep COCs," Theriogenology, vol. 101, pp. 144-150, 2017.

[5] S. S. Chitnis, R. M. Navlakhe, G. C. Shinde et al., "Granulosa cell apoptosis induced by a novel FSH binding inhibitory peptide from human ovarian follicular fluid," Journal of Histochemistry \& Cytochemistry, vol. 56, no. 11, pp. 961-968, 2008.

[6] S. S. Chitnis, C. Selvaakumar, D. D. Jagtap et al., "Interaction of follicle-stimulating hormone (FSH) receptor binding inhibitor8: A novel FSH-binding inhibitor, with FSH and its receptor," Chemical Biology \& Drug Design, vol. 73, no. 6, pp. 637-643, 2009.

[7] T. D. Nandedkar, P. R. Wadia, S. D. Mahale, S. B. Moodbidri, and K. S. Iyer, "Regulation of folliculogenesis by intraovarian peptide," in Follicular growth, ovulation and fertilization: Molecular and clinical basis, A. Kumar and A. K. Mukhopadhyay, Eds., pp. 131-141, Narosa Publishing House, New Delhi, India, 2001.

[8] P. R. Wadia, S. D. Mahale, and T. D. Nandedkar, "Effect of the human follicle-stimulating hormone-binding inhibitor and its $\mathrm{N}$-terminal fragment on follicle-stimulating hormone-induced progesterone secretion by granulosa cells in vitro," Journal of Biosciences, vol. 32, no. 6, pp. 1185-1194, 2007.

[9] S. Mahmood, K. Fahimeh, and Z. Maryam, "Determination of FSH-receptor antagonistic property of 6-amino-4phenyltetrahydroquinoline derivatives by employing QSDAR method," The Journal of Physical Chemistry C, vol. 9, no. 2, pp. $1-6,2014$.

[10] L. Nensi, B. Perin, N. Secen, and D. Sazdanic, "PUB117 Beta Estrogen Receptor as Potential Prognostic Factor in Lung Adenocarcinoma," Journal of Thoracic Oncology, vol. 12, no. 1, pp. S1515-S1516, 2017.

[11] P. Yaşar, G. Ayaz, S. D. User, G. Güpür, and M. Muyan, "Molecular mechanism of estrogen-estrogen receptor signaling," Reproductive Medicine and Biology, vol. 16, no. 1, pp. 4-20, 2017.
[12] C.-L. Lu and C. Herndon, "New roles for neuronal estrogen receptors," Neurogastroenterology \& Motility, vol. 29, no. 7, pp. 11-15, 2017.

[13] P. Rajabi, M. Bagheri, and M. Hani, "Expression of Estrogen Receptor Alpha in Malignant Melanoma," Advanced Biomedical Research, vol. 6, no. 1, p. 14, 2017.

[14] N. Tabatadze and C. Woolley, "Measurement of Inositol Triphosphate Levels from Rat Hippocampal Slices," BioProtocol, vol. 6, no. 7, pp. 20-27, 2016.

[15] D. Neyroud, "Protein fragmentation as a regulatory mechanism: Insights from two different Ca2+ channels, RyR1 and IP3R," Frontiers in Physiology, vol. 7, 2017.

[16] W. Suocheng, G. Zhuandi, A. Lifeng, Z. Taojie, Z. Fengwei, and C. Shien, "Cetrorelix and Triptorelin active immunization influences follicle development and receptor expressions of ovaries in mice," Journal of Applied Biomedicine, vol. 14, no. 1, pp. 49-57, 2016.

[17] S. Wei, S. Chen, Z. Gong et al., "Alarelin active immunization influences expression levels of GnRHR, FSHR and LHR proteins in the ovary and enhances follicular development in ewes," Animal Science Journal, vol. 84, no. 6, pp. 466-475, 2013.

[18] S. Wei, Z. Gong, J. Dong, X. Ouyang, K. Xie, and M. Wei, "Effects of GnRHa active immunity on FSHR expression and uterine development in prepuberty and non cyclic ewes," Iranian Journal of Veterinary Research, vol. 15, no. 3, pp. 244249, 2014.

[19] S. Wei, Z. Gong, L. Sheng, H. Liang, L. Lai, and Y. Deng, "Maturation Rates of Oocytes and Levels of FSHR, LHR and GnRHR of COCs Response to FSH Concentrations in IVM Media for Sheep," Journal of Applied Biomedicin, vol. 15, no. 2, pp. 49-57, 2017.

[20] J. S. Richards, D. L. Russell, S. Ochsner et al., "Novel signaling pathways that control ovarian follicular development, ovulation, and luteinization," Recent Progress in Hormone Research, vol. 57, pp. 195-220, 2002.

[21] C. de Frutos, R. Vicente-Perez, and P. J. Ross, "Determining the requirements for $\mathrm{LH}$ and FSH during sheep in vitro oocyte maturation," Reproduction, Fertility and Development, vol. 26, no. 1, article 200, 2014.

[22] R. M. Navalakhe, D. D. Jagtap, S. U. Nayak, T. D. Nandedkar, and S. D. Mahale, "Effect of FSH receptor-binding inhibitor8 on FSH-mediated granulosa cell signaling and proliferation," Chemical Biology \& Drug Design, vol. 82, no. 2, pp. 178-188, 2013.

[23] R. R. Sullivan, B. R. Faris, D. Eborn, D. M. Grieger, A. G. Cino-Ozuna, and T. G. Rozell, "Follicular expression of follicle stimulating hormone receptor variants in the ewe," Reproductive Biology and Endocrinology, vol. 11, no. 1, pp. 113-116, 2013.

[24] X. Xiao, X. Zi, H. Niu et al., "Effect of addition of FSH, LH and proteasome inhibitor MG132 to in vitro maturation medium on the developmental competence of yak (Bos grunniens) oocytes," Reproductive Biology and Endocrinology, vol. 12, no. 1, pp. 30-31, 2014.

[25] T. R. Monique, G. G. Bernardo, F. I. Gustavo et al., "Expression and molecular onsequences of inhibition of estrogen receptors in granulosa cells of bovine follicles," Journal of Ovarian Research, vol. 7, no. 1, pp. 96-108, 2014.

[26] S. M. Quirk, R. G. Cowan, and R. M. Harman, "The susceptibility of granulosa cells to apoptosis is influenced by oestradiol and the cell cycle," Journal of Endocrinology, vol. 189, no. 3, pp. 441-453, 2006.

[27] X. Liu, D. Qin, Y. Cui et al., "The effect of calcium phosphate nanoparticles on hormone production and apoptosis in human 
granulosa cells," Reproductive Biology and Endocrinology, vol. 8, article no. 32, 2010.

[28] M. Warner, B. Huang, and J.-A. Gustafsson, "Estrogen Receptor $\beta$ as a Pharmaceutical Target," Trends in Pharmacological Sciences, vol. 38, no. 1, pp. 92-99, 2017. 


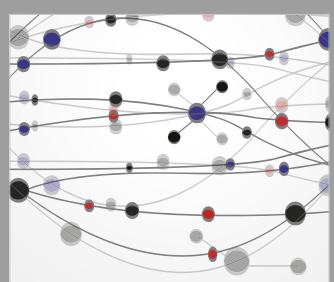

The Scientific World Journal
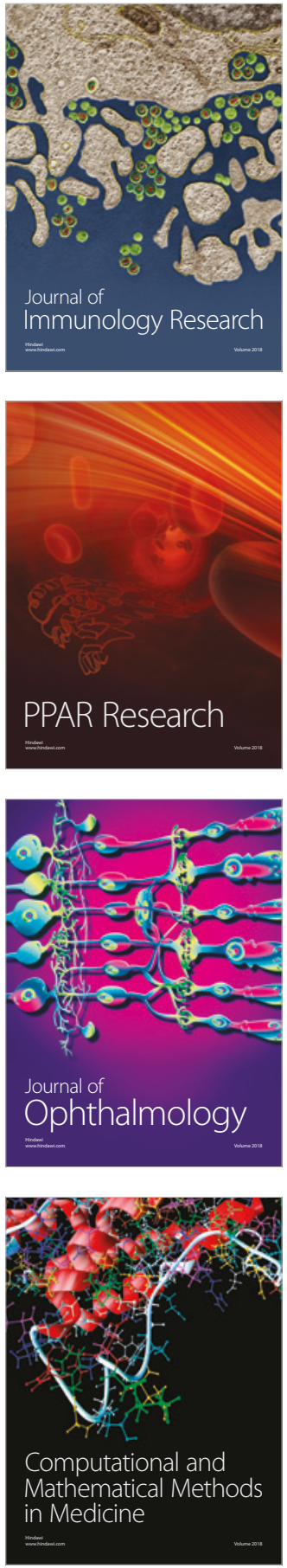

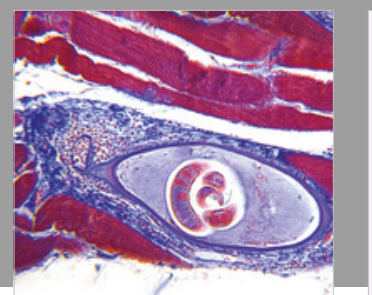

Gastroenterology Research and Practice

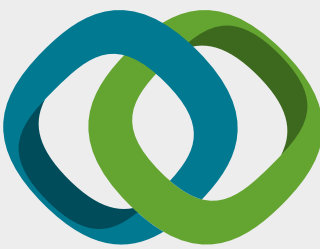

\section{Hindawi}

Submit your manuscripts at

www.hindawi.com
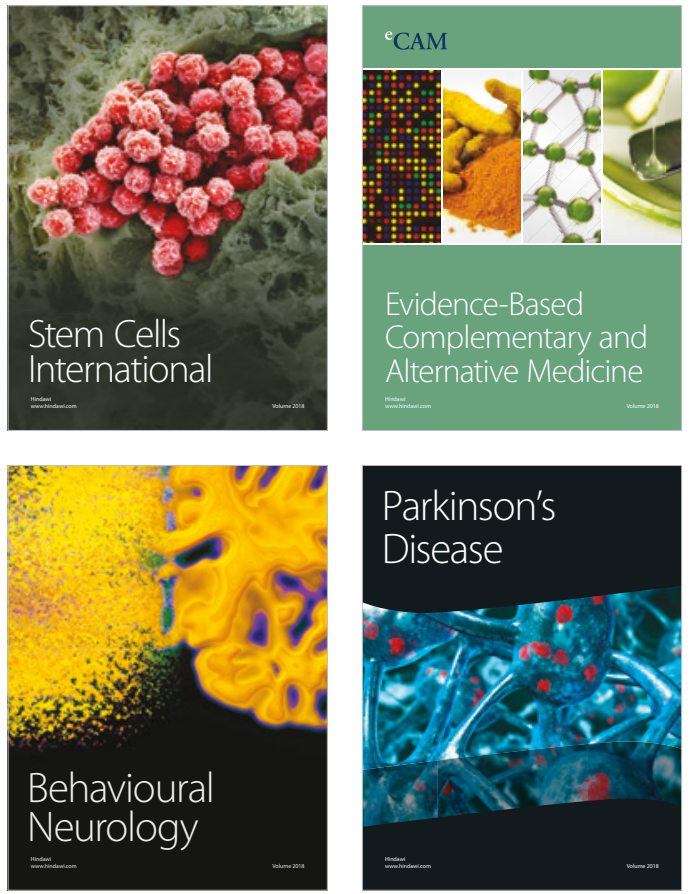

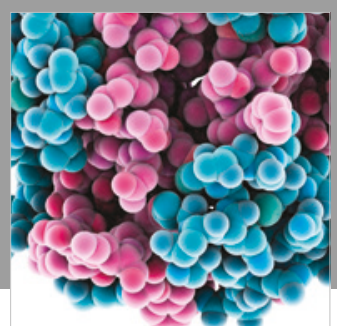

ournal of

Diabetes Research

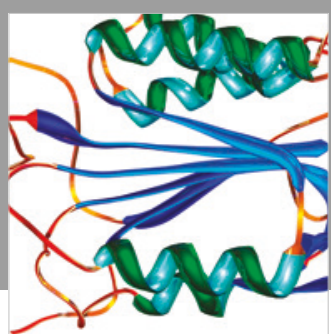

Disease Markers
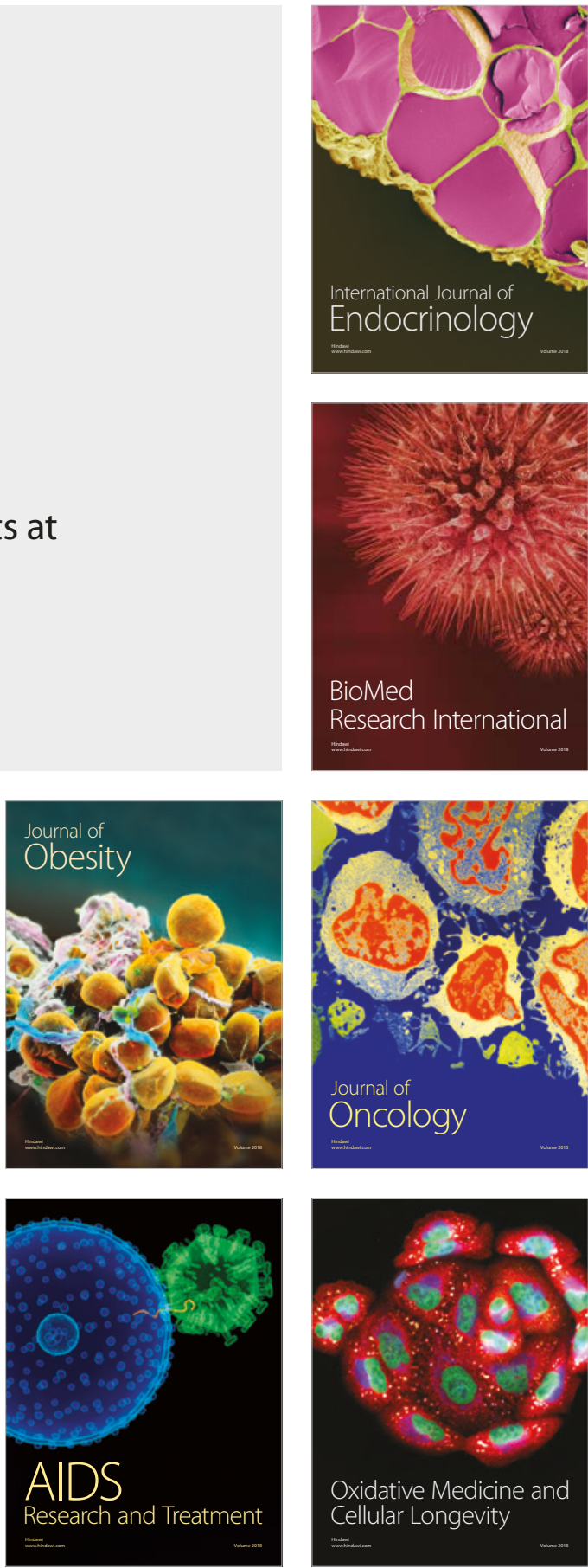